

\title{
Fair User Selection for Zero-Forcing Precoding in Multi-User MISO Systems
}

(C)2009 IEEE. Personal use of this material is permitted. However, permission to reprint/republish this material for advertising or promotional purposes or for creating new collective works for resale or redistribution to servers or lists, or to reuse any copyrighted component of this work in other works must be obtained from the IEEE.

NICOLAS SCHRAMMAR AND PETER A. HOEHER

Stockholm 2010
Communication Theory Department
School of Electrical Engineering
KTH Royal Institute of Technology

IR-EE-KT 2009:064 


\section{Fair User Selection for Zero-Forcing Precoding in Multi-User MISO Systems}

\author{
Nicolas Schrammar \\ School of Electrical Engineering and ACCESS Linnaeus Center \\ KTH, Royal Institute of Technology, Stockholm, Sweden \\ Email: nisc@kth.se
}

\author{
Peter A. Hoeher \\ Faculty of Engineering \\ University of Kiel, Kaiserstr. 2, 24143 Kiel, Germany \\ Email: ph@tf.uni-kiel.de
}

\begin{abstract}
We analyze a multi-user MISO system employing zero-forcing precoding and user scheduling with an adjustable amount of fairness at the transmitter. The system shows a significant gain in sum-rate from multi-user diversity, even for a small number of users. Furthermore, a large fraction of this gain is attained even if the system is constraint to be as fair as the Round-Robin scheduler. The performance of the fair scheduler shows to be unaffected by partial channel state information at the transmitter in the slow fading regime.
\end{abstract}

\section{INTRODUCTION}

Multi-antenna systems have the potential to greatly increase spectral efficiency [1]-[3] and are therefore capable of satisfying the growing demand on data rate of wireless radios. Several strategies to utilize multi-antenna diversity have been proposed. On the one hand dirty paper precoding [4] has been shown to achieve the same scaling as the singleantenna channel, i.e. the rate is unaffected by interference. However, this information theoretic results turns out to be difficult to achieve by practical schemes [5]. On the other hand, zero-forcing (ZF) precoding [6] is a simple straight forward approach canceling interference at the transmitter by multiplying the channel inverse. The main drawback of $\mathrm{ZF}$, noise enhancement, can be overcome if multi-user diversity is inherent in the system, i.e., if the base station can select certain users out of a pool of users. It has been shown that user selection for ZF scales equal to optimal dirty paper coding for large pool sizes [7]. The same scaling can be achieved by greedy user selection [8]. But selecting users opportunistically to maximize sum-rate will result in very much unequal rates provided to the single user. This gives rise to user scheduling under a fairness constraint. Fair user selection has been analyzed for CDMA and OFDM systems in [9] and [10], respectively.

In this paper we analyze a greedy user selection scheme that maximizes the sum-rate of $\mathrm{ZF}$ under an adjustable fairness constraint. Therefore, the scheme exploits the trade-off between rate and fairness. The fairness is measured using two different fairness metrics. The objective is to find the sum-rate of a system that is equally fair as the Round-Robin scheduler [11]. Furthermore we analyze the system in the realistic scenario, where the base station obtains channel state information (CSI) only from those users that were selected. The resulting sum-rate is found for different fading conditions.
The remainder of the paper is organized as follows. In section II we present the system and channel model as well as the precoding strategy. The throughput of the system is derived. Section III introduces the scheduler with adjustable fairness constraint. In section IV we present and discuss the simulation results, and section $\mathrm{V}$ concludes the paper.

\section{FUnDAMENTALS}

\section{A. System Model}

We consider the downlink transmission from a base station equipped with $M$ antennas to $K$ mobile stations (users) each equipped with one antenna. The transmission over the wireless medium is modeled as

$$
y_{i}(t)=\boldsymbol{h}_{i}^{T}(t) \boldsymbol{s}(t)+n_{i}(t), \quad i \in\{1, \ldots, K\} .
$$

Subsequently, we drop the time index $t$ for ease of notation. The vector of transmitted signals and the $i$-th user's received signal are denoted as $s \in \mathbb{C}^{M}$ and $y_{i} \in \mathbb{C}$, respectively. The noise $n_{i} \sim \mathcal{C N}(0,1)$ is complex Gaussian, and the power of transmitted signal is constrained to $\|s\|^{2}=\rho$. The user's channel vectors $\boldsymbol{h}_{i} \in \mathbb{C}^{M}$ form the channel matrix $\boldsymbol{H}=\left[\boldsymbol{h}_{1}^{T} \ldots \boldsymbol{h}_{K}^{T}\right]^{T}$, that yields the equivalent channel model

$$
\boldsymbol{y}=\boldsymbol{H} \boldsymbol{s}+\boldsymbol{n},
$$

with $\boldsymbol{y}=\left[y_{1} \ldots y_{K}\right]$ and $\boldsymbol{n}=\left[n_{1} \ldots n_{K}\right]$. The entries of $\boldsymbol{H}$ are i.i.d zero-mean unit-variance complex Gaussian $h_{i, j} \sim \mathcal{C N}(0,1), j \in\{1, \ldots, M\}$ and are time correlated. The correlation is modeled by Rayleigh fading with maximum Doppler shift $f_{D}$, and the channel is assumed to stay constant for transmission blocks of length $T_{b}$.

\section{B. Precoding}

Since the users cannot decode the received signals jointly, the interference caused by the channel must be compensated at the transmitter. One simple way of achieving this is linear ZF precoding, that is multiplication by the Moore-Penrose pseudo inverse

$$
\boldsymbol{P}=\boldsymbol{H}^{H}\left(\boldsymbol{H} \boldsymbol{H}^{H}\right)^{-1}
$$

at the transmitter, i.e.

$$
s=\sqrt{\rho} \frac{\boldsymbol{P a}}{\|\boldsymbol{P a}\|} .
$$


The vector $\boldsymbol{a} \in \mathbb{C}^{K}$ contains the symbols intended for the users. Plugging into (1) yields

$$
\boldsymbol{y}=\frac{\sqrt{\rho}}{\|\boldsymbol{P a}\|} \boldsymbol{a}+\boldsymbol{n} .
$$

We observe that each user's received symbol $y_{i}$ is a scaled and noisy version of the intended symbol $a_{i}$.

\section{Sum-rate}

According to [12] the sum-rate of a system using $\mathrm{ZF}$ is

$$
R^{\mathrm{wf}}=\sum_{i=1}^{K}\left[\log _{2}\left(\mu b_{i}\right)\right]^{+}
$$

with the coefficients

$$
b_{i}=\frac{1}{\left[\left(\boldsymbol{H} \boldsymbol{H}^{H}\right)^{-1}\right]_{i, i}}
$$

The water-level $\mu$ is obtained from the water-filling formulation

$$
\rho=\sum_{i=1}^{K} \rho_{i}=\sum_{i=1}^{K}\left[\mu-\frac{1}{b_{i}}\right]^{+}
$$

with power constraint $\rho$ and $[x]^{+}=\max (0, x)$.

The procedure of water-filling will be omitted in this paper, because it conflicts with fairness constraints. Fairness requires selecting users with low channel gain, but water-filling will possibly assign no transmit power to those users. Having no constant water-level we get $\mu_{i}=\rho_{i}+\frac{1}{b_{i}}$ and therefore

$$
R=\sum_{i=1}^{K} \log _{2}\left(1+\rho_{i} b_{i}\right)=K \log _{2}(1+\vartheta) .
$$

The second relation follows from the fact that without waterfilling all users experience the same signal-to-noise ratio (SNR) $\vartheta$. From (3) and (2) we find

$$
\vartheta=\frac{\rho}{\sum_{i=1}^{K} \frac{1}{b_{i}}}=\frac{\rho}{\operatorname{tr}\left(\left(\boldsymbol{H} \boldsymbol{H}^{H}\right)^{-1}\right)}=\frac{\rho}{\left\|\boldsymbol{H}^{\dagger}\right\|_{\mathrm{F}}^{2}},
$$

where the last relation is due to the definition of pseudo-inverse and of Frobenius-norm. Now the sum-rate becomes

$$
R=K \log _{2}\left(1+\frac{\rho}{\|\boldsymbol{P}\|_{\mathrm{F}}^{2}}\right)
$$

In fact we have a channel dependent SNR degradation $\|\boldsymbol{P}\|_{\mathrm{F}}^{2}$. The degradation depends on the condition of the channel matrix $\boldsymbol{H}$. An ill-conditioned matrix results in an inverse with large entries. Multi-user diversity enables the scheduler to choose a subset of users that possesses a channel matrix with better condition and hence increased sum-rate.

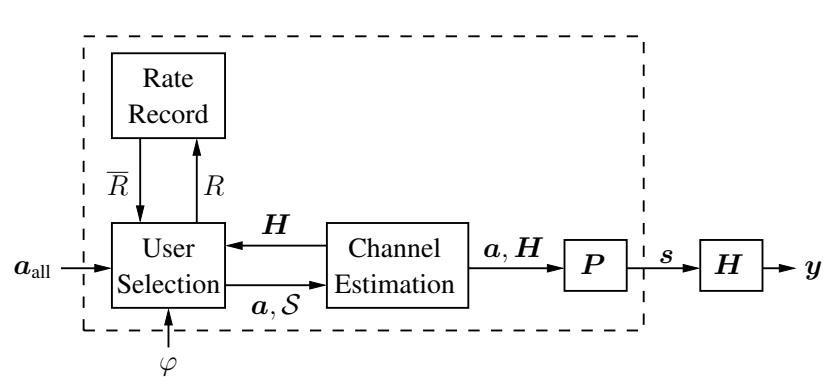

Fig. 1. Block diagram of communication system.

\section{FAIR USER SELECTION}

We focus on greedy user selection due to its low complexity and high performance compared to optimal exhaustive search user selection [8]. The scheduler selects $K=M$ users out of the pool of $N$ users in a successive manner. If no fairness constraint is imposed, user selection maximizes the sum-rate $R$ or equivalently minimizes $\|\boldsymbol{P}\|_{\mathrm{F}}^{2}$.

Suppose a set $\mathcal{S}$ of users has been selected in previous selection steps and a disjoint set $\mathcal{R}$ contains the users that were not selected, yet. Then greedy user selection can be expressed as

$$
i=\arg \min _{i^{\prime} \in \mathcal{R}}\left\|\boldsymbol{P}\left(\mathcal{S} \cup\left\{i^{\prime}\right\}\right)\right\|_{\mathrm{F}}^{2},
$$

where $\boldsymbol{P}(\mathcal{A})$ is the pseudo-inverse of the channel matrix $\boldsymbol{H}(\mathcal{A})$ consisting of the channel vectors $\boldsymbol{h}_{i}, i \in \mathcal{A}$ of the selected users.

To incorporate fairness, we modify the minimization according to

$$
i=\arg \min _{i^{\prime} \in \mathcal{R}}\left(\left\|\boldsymbol{P}\left(\mathcal{S} \cup\left\{i^{\prime}\right\}\right)\right\|_{\mathrm{F}}^{2} \cdot \bar{R}\left(i^{\prime}\right)^{\varphi}\right),
$$

where the $i$-th user's mean rate $\bar{R}(i)$ acts as a penalty multiplier. The higher the rate that user received in the past, the more unlikely it will be selected. The mean rate is obtained from exponentially weighted moving averaging over the instantaneous rate. The fairness exponent $\varphi$ governs the amount of fairness in weighting the penalty multiplier. The block diagram of the communication system is depicted in Fig. 1.

To determine the amount of fairness in the system we use Jain's fairness metric [13]

$$
F_{\mathrm{J}}=\frac{\left(\sum_{i=1}^{N} \bar{R}(i)\right)^{2}}{N \sum_{i=1}^{N}(\bar{R}(i))^{2}},
$$

where $N$ is the total number of users, as well the simple minmax-metric

$$
F_{\mathrm{mm}}=\frac{\min _{i} \bar{R}(i)}{\max _{i} \bar{R}(i)}
$$




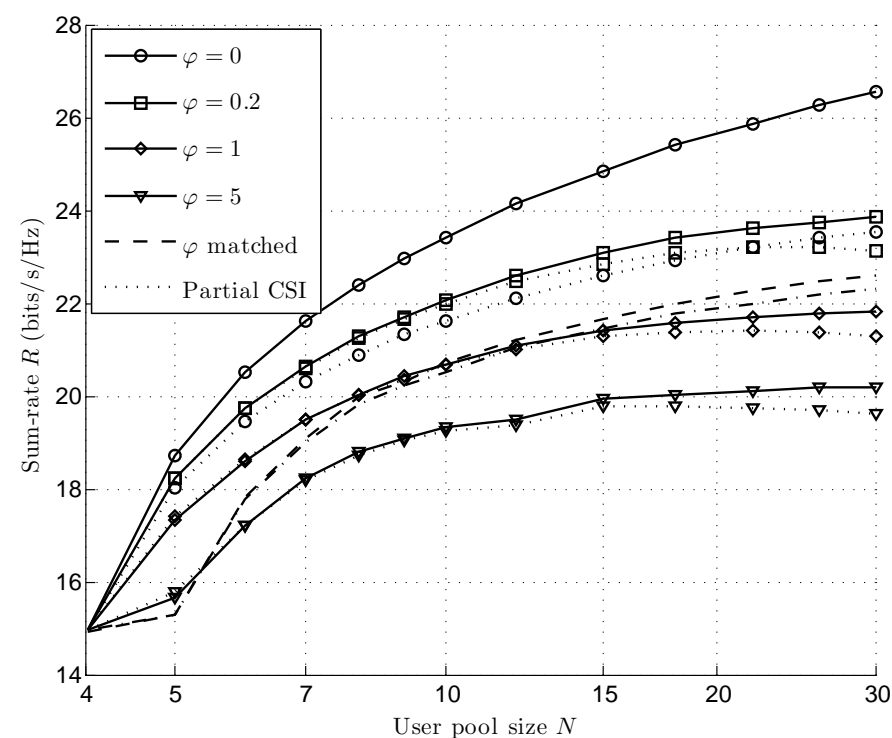

Fig. 2. Sum-rate vs. number of users in pool for $N=4$ antennas and normalized fade rate $f_{n}=1 / 200$.

\section{REsults}

The objective of our work is to find the sum-rate $R$ subject to the fairness exponent $\varphi$, i.e. how much of the gain from user selection can be preserved under different fairness constraints. We fix the $\operatorname{SNR} \rho=20 \mathrm{~dB}$, since for $\rho \gg 1$ it acts merely as an offset in (4). The realizations of the Rayleigh fading channel are generated using Jakes' model [14] with normalized fade rate $f_{n}=f_{D} T_{b}$, the measure of channel variation. The maximum maximum Doppler shift $f_{D}=v f_{c} / c$ is a function of carrier frequency $f_{c}$, speed of mobile stations $v$ and speed of light $c$.

Fig. 2 shows the sum-rate $R$ as a function of the number of users $N$ in the system. The normalized fade rate is $f_{n}=1 / 200$, the number of transmit antennas is $M=4$ and we select $K=4$ users in each transmission block. First, consider the solid lines, as they show the dependence on the fairness exponent $\varphi$. The value of $\varphi$ is indicated by different markers. We can observe a graceful degradation in sum-rate with increasing fairness exponent. However, the gain from multi-user diversity is still significant. Furthermore, the main part of the gain is due to the first additional users in the pool. This desirable property is still attained under the fairness constraint.

An important question is, what value of $\varphi$ will result in an amount of fairness that is sufficient for the system. To answer this question we compare our scheme to a RoundRobin user selection. This scheduler selects the users in a specific sequence, i.e. perfectly fair. We measure the Jainfairness using the metric (5) for each value of $N$. Secondly we search for the fairness exponent $\varphi$ of our scheduler, that reaches the same Jain-fairness as the Round-Robin scheduler. Finally, we simulate the sum-rate for the matched fairness exponents. The result is indicated by the dashed line without marker in Fig. 2. To check whether the result is dependent

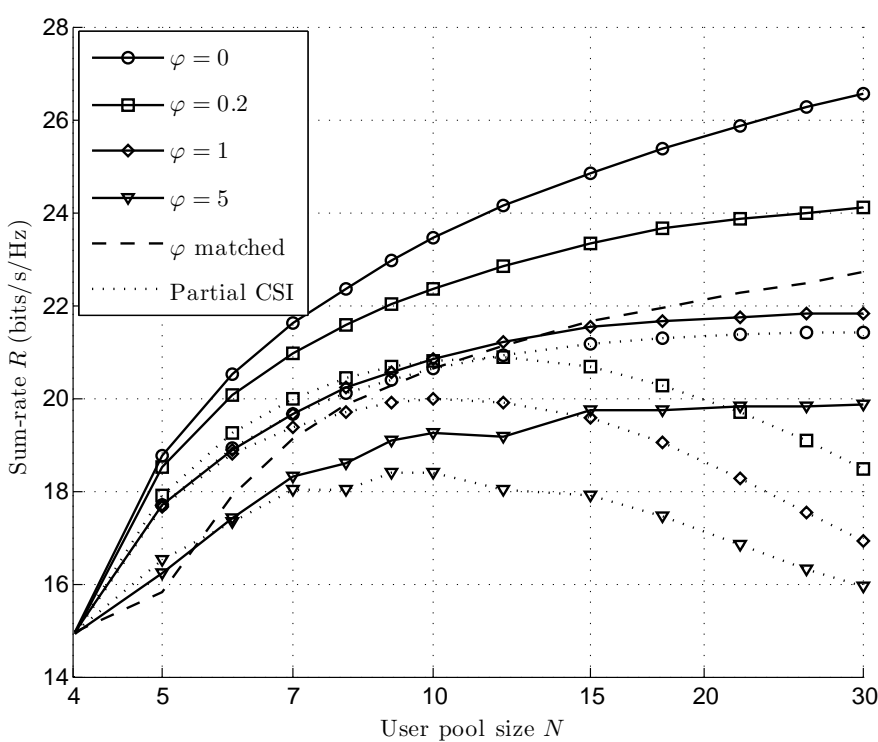

Fig. 3. Sum-rate vs. number of users in pool for $N=4$ antennas and normalized fade rate $f_{n}=1 / 50$.

on the fairness metric, the simulation is repeated using the min-max metric (6) instead. The dash-dotted line suggests that the result is almost independent of the metric. For a fairness exponent around $\varphi=1$ our scheduler is as fair as the reference scheduler.

Surprisingly, our user selection scheme gains much sumrate even if it is constrained to be as fair as the Round-Robin scheduler. This is our most important result, it indicates that even under strong fairness constraints user selection should be optimized to maximize sum-rate.

Next, we investigate to what extend our scheduler is dependent on accurate CSI of all user in the pool. For large pool sizes $N$ it may be expensive or impossible to acquire CSI from all users in each transmission block. Instead we assume the base station attains CSI only from those users that have been selected. Therefore scheduling is based on partially outdated CSI. The dotted lines in Fig. 2 show the resulting sum-rates for different fairness exponents $\varphi$ indicated by different markers. First of all, unfair user selection $(\varphi=0$ marked with circles) shows a large degradation, but as soon as fairness constraints are included the degradation vanishes, the lines almost coincide. Fair user selection results in more accurate CSI, because deselecting a user for a long period of time is avoided.

Turning to Fig. 3 we observe the dependence on the fade rate $f_{n}$ of the channel. The system was simulated with the same parameters but with faster fading $f_{n}=1 / 50$. Firstly, the sum-rate shows a similar dependence on the fairness exponent under the assumption of perfect CSI (solid lines). Secondly, the statement about the sum-rate under fairness constraint dictated by Round-Robin scheduling still holds (dashed line). However, the performance is degraded under the partial CSI assumption (dotted lines), and the higher the number of users $N$, the higher the degradation. This is reasonable, since if there are many 


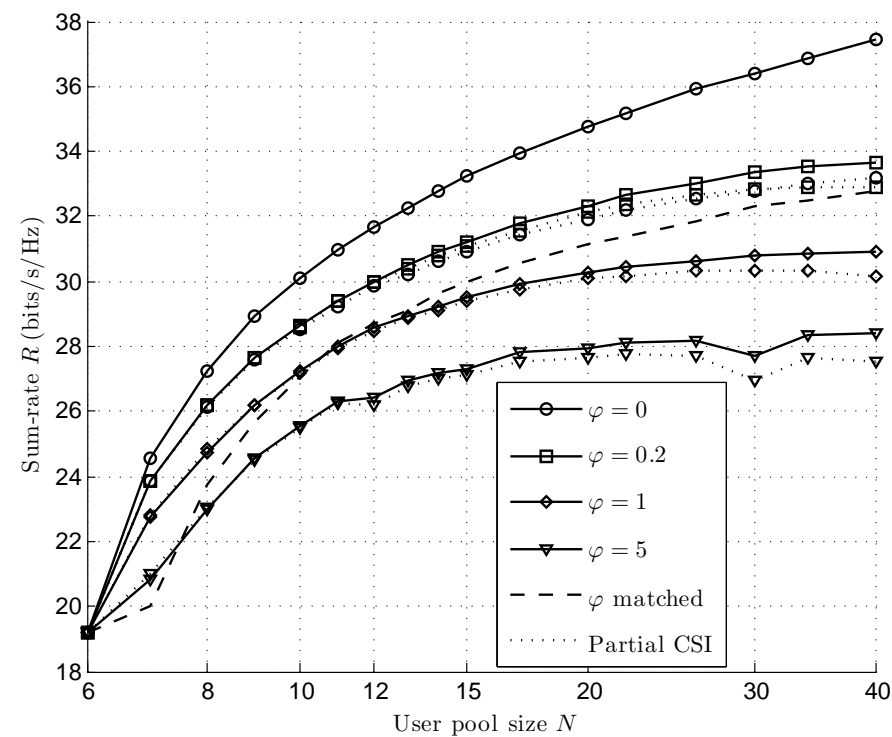

Fig. 4. Sum-rate vs. number of users in pool for $N=6$ antennas and normalized fade rate $f_{n}=1 / 200$.

users competing for transmission, the base station's CSI will be less accurate. Accordingly, the number of users $N$ should be matched to the fade rate, if only partial CSI is available. In the slow fading regime, though, the transmitter is able to keep track of a large amount of users $N$ (see Fig. 2).

Finally, Fig. 4 and Fig. 5 show similar results for $M=$ 6 and $M=8$ receive antennas in the slow fading regime $f_{n}=1 / 100$, respectively. A scheduler, that is equally fair as the Round-Robin scheduler employs a fairness exponent around $\varphi=1$. This suggests that the choice of the fairness exponent can be made independent of the system dimension. With increasing number of antennas $M$, the receiver is more robust against partial CSI (compare dotted lines in Fig. 2, Fig. 4 and Fig. 5).

\section{CONCLUSION}

We presented results from simulating a multi-user MISO system employing a scheduler with adjustable fairness. We found that the diversity gain in sum-rate can be achieved even under a strict fairness constraint, forcing the system to be as fair as the Round-Robin scheduler. Since Round-Robin treats the users with maximum fairness, our results indicate, that opportunistic scheduling is beneficial under every fairness constraint. The performance degradation of user selection under realistic, i.e. partial CSI is alleviated by the fair scheduler due to shorter periods of deselection and hence more accurate CSI.

\section{ACKNOWLEDGMENT}

This work is based upon the master thesis of N.S. at University of Kiel, Germany in cooperation with Toshiba Telecommunications Research Laboratory, Bristol, UK. The author thanks Joe McGeehan, Vishakan Ponnampalam and Magnus Sandell of Toshiba TRL for their support and guidance.

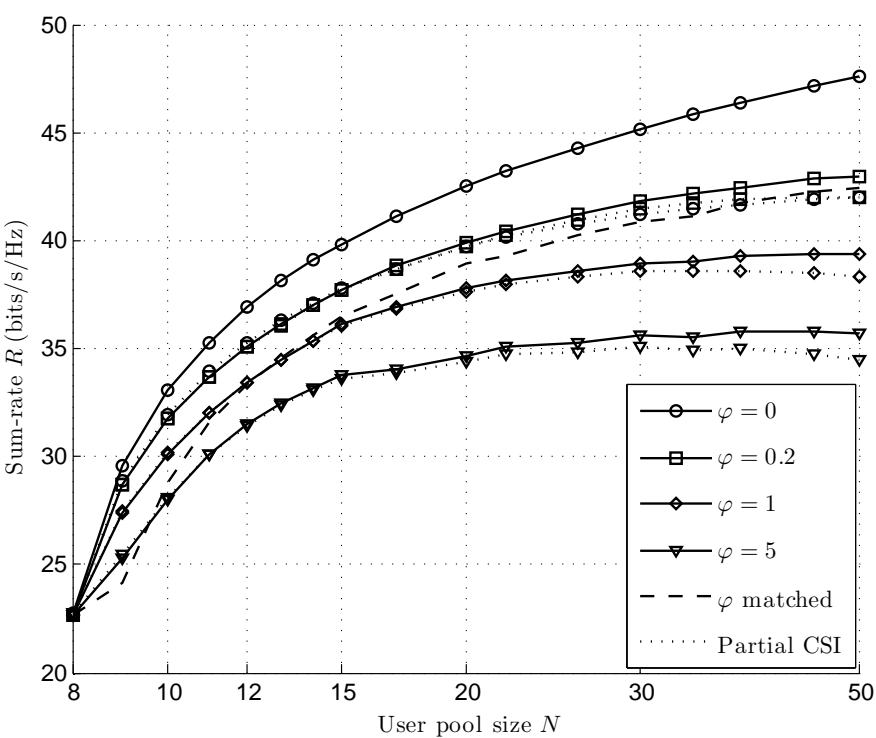

Fig. 5. Sum-rate vs. number of users in pool for $N=8$ antennas and normalized fade rate $f_{n}=1 / 200$.

\section{REFERENCES}

[1] G. J. Foshini and M. J. Gans, "On limits of wireless communications in a fading environment when using multiple antennas," Wireless Personal Communications, pp. 311-335, Mar. 1998.

[2] E. Telatar, "Capacity of multi-antenna Gaussian channels," European Transactions on Telecommunications, vol. 10, no. 6, pp. 585-596, Nov. 1999.

[3] J. Mietzner, R. Schober, L. Lampe, W. H. Gerstacker, and P. A. Hoeher, "Multiple-antenna techniques for wireless communications a comprehensive literature survey," IEEE Communications Surveys \& Tutorials, vol. 11, no. 2, pp. 87-105, 2009.

[4] M. H. M. Costa, "Writing on dirty paper," IEEE Transactions Information Theory, vol. IT-29, no. 3, pp. 439-441, May 1983.

[5] M. Airy, A. Forenza, J. Heath, R.W., and S. Shakkottai, "Practical costa precoding for the multiple antenna broadcast channel," in GLOBECOM '04. IEEE, vol. 6, 2004, pp. 3942-3946.

[6] A. Wiesel, Y. C. Eldar, and S. Shamai, "Zero-forcing precoding and generalized inverses," IEEE Transactions on Signal Processessing, vol. 56, no. 9, pp. 4409-4418, 2008.

[7] T. Yoo and A. Goldsmith, "On the optimality of multiantenna broadcast scheduling using zero-forcing beamforming," IEEE Journal on Selected Areas in Communications, vol. 24, no. 3, pp. 528-541, 2006.

[8] J. Wang, D. J. Love, and M. D. Zoltowski, "User selection with zeroforcing beamforming achieves the asymptotically optimal sum rate," IEEE Transactions on Signal Processessing, vol. 56, no. 8, pp. 37133726, 2008.

[9] E. Lim and S. Kim, "Transmission rate scheduling with fairness constraints in downlink of CDMA data networks," IEEE Transactions on Vehicular Technology, vol. 54, no. 1, pp. 328-337, 2005.

[10] Z. Zhang, Y. He, and E. K. P. Chong, "Opportunistic downlink scheduling for multiuser OFDM systems," in Proc. Wireless Comm. and Netw. Conf., vol. 2, 2005, pp. 1206-1212.

[11] F. Cottet, J. Delacroix, C. Kaiser, and Z. Mammeri, Scheduling in RealTime Systems. Wiley, 2002.

[12] G. Caire and S. Shamai, "On the achievable throughput of a multiantenna Gaussian broadcast channel," IEEE Transactions on Information Theory, vol. 49, no. 7, pp. 1691-1706, Jul. 2003.

[13] R. K. Jain, D.-M. W. Chiu, and W. R. Hawe, "A quantitative measure of fairness and discrimination for resource allocation in shared computer systems," DEC Research Report, vol. TR-301, Sep. 1984.

[14] W. C. Jakes, Ed., Microwave Mobile Communications. Wiley, 1974. 